\title{
Measuring change and changing measures: The development of a torture survivor specific measure of change
}

\author{
Rebecca Horn, PhD, Andy Keefe*
}

\begin{abstract}
Freedom from Torture is a UK-based human rights organisation dedicated to the treatment and rehabilitation of torture survivors. The organisation has been working towards the development of a clinical outcome tool for a number of years, and the purpose of this paper is to (a) describe the process of developing the tool and the final tool itself, and (b) to outline the system which Freedom from Torture has established to collect, record and analyse the data produced.

A review of the literature revealed that existing measures were not appropriate for measuring psychological and emotional change amongst torture survivors; therefore the organisation undertook to develop a tool specifically designed for this target group. The clinical outcome tool was developed collaboratively by Freedom from Torture clinicians, clients, interpreters and an external consultant. Initial discussions took place with clinicians and clients to develop an understanding of what psychosocial wellbeing and psychosocial distress meant to this unique population of torture survivors, and which issues and features should be
\end{abstract}

\footnotetext{
^) Freedom from Torture, London, UK

Correspondence to: akeefe@freedomfromtorture.org
}

included in the clinical outcome tool. A process of discussion and testing of potential approaches led to the development of a draft clinical outcome tool which was translated into 15 languages and then pilot tested with 151 clients.

The data from the pilot study was analysed and used to produce the final version of the clinical outcome tool. The clinical outcome tool was formally rolled out across the organisation's five centres in April 2014. Clinicians working with adult clients have been completing it at the beginning of therapy and then again at regular intervals.

The data from the first year is currently being analysed, and the experiences of clinicians, clients and interpreters of using the clinical outcome tool are being reviewed, with a view to continuing to develop and improve the tool and the processes by which it is used. Ultimately, the data will be used to improve the services offered to survivors of torture in the UK.

Keywords: outcome measures, torture, psychology, mental health

\section{Introduction}

Freedom from Torture has been supporting torture survivors for 30 years and is the only UK-based human rights organisation dedicated to the treatment and rehabilitation 
of torture survivors. The organisation offers services to around 1,000 survivors of torture per year from its centres in London, Birmingham, Manchester, Newcastle and Glasgow. Services include psychological, and physical therapies, forensic documentation of torture, legal and welfare advice, and creative projects. Together with survivors, the organisation builds the capacity of the judiciary and health, social and education services to respond to the needs of survivors of torture, advocates for improvements in the conditions of survivors in the UK and holds torturing states to account through advocacy with international bodies, such as the United Nations through its Country Reporting Programme.

The organisation has been working towards the development of a clinical outcome tool for a number of years, and the purpose of this paper is to (a) describe the process of developing the tool and the final tool itself, and (b) to outline the system which Freedom from Torture has established to collect, record and analyse the data produced.

The trustees, managers and practitioners within Freedom from Torture recognise the need to assess the ways in which clients' wellbeing is influenced by their engagement with Freedom from Torture services.

Essentially, it is an "exercise in accountability" (p114) ${ }^{1}$ to all stakeholders, including the survivors who engage with Freedom from Torture, donors and the wider community of organisations providing services to survivors of torture. Ethically, it is essential to take measures to assess whether the interventions offered have a positive impact on wellbeing, and to have some understanding of which components of interventions are effective with different populations, to assist with planning and ensure that clients receive the help they need. ${ }^{1,2}$ Assessments are also necessary to ensure that resources are well-used; this is particularly important in times of financial constraint.

\section{Brief review of other outcome research with survivors of torture}

A number of reviews have been published of outcome research conducted with survivors of torture. ${ }^{2,3,4}$ These reviews have included studies conducted in a wide variety of environments, including refugee camps, the countries in which the torture took place, and countries which survivors have fled to in order to seek safety. Since Freedom from Torture's work is with torture survivors who have come to the UK to seek safety and protection, this discussion will focus mainly on outcome research conducted with that population.

There is a general consensus in the literature that there has been little research into the outcomes of psychological services for torture survivors, and much of that which has been conducted is flawed. The main limitations are outlined below.

\section{Too narrow a range of outcome measures/} indicators of wellbeing have been used: This is the weakness cited most often in relation to outcome studies conducted with torture survivors. ${ }^{3,4,5}$ The wide range of ways in which torture impacts on subsequent wellbeing has been noted by many authors, and includes 'inadequate social support, interpersonal and family relationships; psychological problems including shame, guilt, low self-esteem and feeling disempowered; and those related to lacking agency and control in one's life, having lost a sense of meaning, purpose and worth as a human being and having no sense of justice. ${ }^{4}$ Yet the majority of outcome studies focus on mental health, particularly symptoms of post-trau- 
matic stress disorder (PTSD). These issues, whilst relevant in many cases, may not be the most meaningful to the torture survivors themselves. In addition, they may not capture treatment goals or the range of ways in which interventions can impact on survivors' lives. ${ }^{4}$

\section{Studies neglect the contexts in which survivors} live, and the impact of daily stressors on their wellbeing: Related to the above, many studies do not take into account the ongoing daily stressors affecting the wellbeing of those engaging in therapy, ${ }^{1,3}$ such as news from home, the process of applying for refugee status, housing issues and physical health. This neglect makes it difficult to understand the ways in which the therapeutic process interacts with the many other influences on clients' wellbeing. Events such as bad news from home can halt or even reverse progress in therapy, and so affect outcomes. "These kinds of ecological factors are likely to influence treatment outcomes but are rarely considered in research designs" (p.559). ${ }^{3}$

\section{Assessment/measurement instruments are not} validated with this group, and/or are not suitable for this population: This is another common limitation of outcome studies with torture survivors. It is related to the first limitation outlined here, in that tools are often diagnosis-based and have been developed for use with a western population. The concepts on which the tools are based may not be meaningful outside this context, which would impact on their validity with other populations. In a number of studies, tools were used without their validity being assessed with the target population. ${ }^{4} \mathrm{~A}$ specific problem related to the use of symptom-based tools not developed for use with torture survivors is the 'ceiling effect'. ${ }^{3}$ If the symptom level reported by a client is so high that it reaches the maximum level of severity it is difficult to measure improvement; even if the client reports that they feel better, they may still report the highest level of severity on the scale.

\section{Translation of tools has not been conducted} adequately: In some studies, tools were not assessed for conceptual validity before translation; in others the measures were translated but their validity was not evaluated; and in some cases interpreters translated the questions 'in real time' during the research interviews, introducing variation in the use of terms and response options. ${ }^{4}$

Lack of control groups: Without a control group it can be difficult to understand the extent to which any changes observed are due to the intervention. Whilst there are challenges to conducting randomised controlled trials to assess the effectiveness of services for torture survivors, there are alternatives which can be used to understand the impact of particular services or combinations of services on wellbeing, but few outcome studies have adopted these. ${ }^{2,4}$

Small sample sizes: Many studies have sample sizes which are too small to enable comparisons to be made between different groups. ${ }^{2,3,4,5}$

Insufficient comparison of treatment effectiveness of survivors from different cultural backgrounds, and with different experiences: One of the consequences of small sample sizes is that it is difficult or impossible to compare the ways that different groups of survivors respond to different forms of intervention. There is great diversity amongst torture survivors in the UK, for example, in terms of life history, cultural background, world outlook, experience of torture, and post-displacement 
experiences, and very few studies have been able to draw conclusions about treatment effectiveness for different groups of survivors. McFarland and Kaplan ${ }^{3}$ note that the literature suggests that receptivity and response to intervention type is influenced by the cultural and ethnic background of both the recipient and the therapist, and that questions remain about the relative effectiveness of different treatments for people of different cultural backgrounds ( $\mathrm{p} 557$ ).

The issue of diversity, both of torture survivors and the services offered, is a significant challenge to those conducting outcome studies. ${ }^{2,3,5}$ Very large sample sizes are required in order to understand the impact on outcomes of the many different client and treatment variables, and in most studies it has not been possible to obtain sufficient samples to explore these issues.

An additional challenge is that many torture survivors require therapy over a long period of time, and their progress is not linear. It can be set back by, for example, news from home, a disappointing letter regarding their asylum process or any factor that reminds them of previous distressing events. This can create a confusing picture, especially if contextual factors are not included in the outcome measure, and/or if sample sizes are small, and/or if the data is collected over a relatively short period of time. ${ }^{2}$

Characteristics of good research into the outcomes of interventions for torture survivors

The review of the literature indicated that no measurement instruments were available with unequivocal relevance for survivors of torture. However, drawing from the reviews referred to above, we can develop a summary of the features of studies that are likely to help us to understand the effectiveness of interventions with this population. These features are:
- Measurement tools and approaches should consider the context within which torture survivors live (e.g. as an asylum seeker or refugee), and the impact of this context on their wellbeing. ${ }^{1,3,4}$ External factors can have a significant influence, both positive and negative, on wellbeing.

- A broad range of outcomes should be considered, prioritising those which have the greatest meaning and relevance for the torture survivors themselves. ${ }^{1,3,4}$

- Clinicians and other practitioners should be involved in the identification of outcomes and development of questions to be included in a measurement tool. ${ }^{3}$

- Translators should be involved in the development of questions to be included in a measurement tool, to strengthen cultural appropriateness and conceptual validity. ${ }^{4}$

-Where the population is heterogeneous (i.e. there is high variation on a number of variables), the sample size should be large enough to make comparisons. ${ }^{3,5}$ For example, exploring the effectiveness of different treatment options for people with different types of experiences (pre and post displacement - e.g. asylum status), and from different cultural backgrounds.

- Outcomes should be assessed over a longer period than has been the case in previous studies. ${ }^{3,5}$

We would also suggest that, since survivors of torture typically suffer from multiple psychological/psychiatric, somatic and social problems and may have experienced repeated interrogations, it is necessary for measurement tools to be as brief as possible and to include opportunities for explanation and discussion, rather than a 'checklist' type format, in order to avoid exacerbating distress.

A further key characteristic of good outcome research is that it is conducted in 
an organisation which values and 'mainstreams' assessment, so that it is not seen as an 'add-on', but as a fundamental part of its ongoing work A task force of senior clinicians from the United States was organised to develop quality assurance criteria for torture treatment centres. This task force recommended several principles:

1) Measuring outcomes should be an integral part of the care.

2) Practically, assessment must be integrated into the daily routine of the programme, not as separate research.

3) Outcome measurement should be part of a process which includes analysis and reporting of the data and improving the quality of care through education and training of the providers."(p.105). ${ }^{2}$

Patel and Williams ${ }^{1}$ state that " [i]t is only by routine measurement of all clients ... that a service can start to answer important questions: what gains are clients making? What size are those gains, at best, on average and at least? Do some clients get worse? It can even address questions such as: do longer treatments bring about greater changes than shorter, or do gains tend to level off?"(p107)

Organisational commitment to the process of assessing outcomes, and using that information to improve services, requires the allocation of resources. This may include hiring specialist staff for certain aspects of the process, incorporating tasks related to outcome measurement in the job descriptions of various staff members, including clinicians who collect and (sometimes) enter the data, and allowing time for these tasks. Time and resources must also be allocated to tasks related to developing cross-culturally validated translations of the tool. The development and maintenance of a data recording system requires IT expertise, and the data analysis requires specialist skills, all of which may have resource implications. It is essential, therefore, that an organisation plans for all these stages and is fully committed to the process before beginning.

In 'mainstreaming' assessment of clients' progress and outcomes, it is important that both the outcome tool and the process by which it is used is accepted by both clinicians and clients, ${ }^{2}$ as well as interpreters, who play a key role in generating an atmosphere of safety. Patel and Williams ${ }^{3}$ note the many pressures which clinicians working with torture survivors face, and the anxiety which can be triggered when an additional, potentially burdensome, task is introduced. For some clinicians, the introduction of an outcome tool can raise concerns about its purpose and how the information will be used. If clinicians perceive the assessment tool and process as excessively burdensome and/or threatening, there will be a negative impact on the data obtained. It is essential, therefore, to develop an assessment tool and process in a way which minimises the burden on staff and is perceived to have both immediate and longer-term benefits for both clinicians and clients. Patel and Williams also emphasise the importance of ensuring that the assessment tool and process is respectful of clients: they "need to feel that their views are respected, that their responses are valued, and that practitioners see them and treat them as human beings, not as objects from which to extract information required by the organisation" (p64). ${ }^{1}$ Clients must feel they can be honest without the fear of negative consequences, and must understand what the information is for, and how it will be stored and used.

This brief review of the field illustrates the many challenges involved in measuring the outcomes of interventions with torture survivors, but also how important it is to start doing so, even in an imperfect way. 
This is expressed well by Jaranson and Quiroga ${ }^{2}$ who write: "Perhaps most importantly, centres must start collecting data. Even if only descriptive or demographic data is available, this data should be collected ... When developing an outcome study, it is important to remember a caveat by Voltaire: 'The best is the enemy of the good.' Even if you can't do an evaluation that meets all of the scientific criteria for perfection, please start doing something"(p133).

In this paper we share the clinical outcome tool developed by Freedom from Torture, and the process by which it is being used, not as an example of a perfect solution to the challenge of assessing the effectiveness of interventions with torture survivors, but as a work in progress which we will continue to develop and improve as it is put into practice.

\section{Context and approach}

The aim of the project was to develop a clinical outcome tool which could be used to understand the ways in which adult clients change through their engagement with individual psychological therapy offered by Freedom from Torture. This information would be used at an organisational level to demonstrate to outside bodies (funders, partners, organisations receiving training) the effects of the approach taken by Freedom from Torture with this client group.

The questions which data from the clinical outcome tool could be used to answer include:

- How do clients change as they participate in therapy?

- How are clients different at the end of therapy compared to the beginning?

-What external factors contribute to or hinder progress in therapy?

-What aspects of the therapeutic process do clients find most helpful?
In the spirit of 'starting small' advocated by Jaranson and Quiroga, ${ }^{2}$ it was planned to begin by developing a tool suitable for use with adults participating in individual psychological therapy. Once this tool had been successfully developed, and it was being used, it would then be possible to consider adapting it for use with young people, clients participating in groupwork, and so on.

The level of organisational commitment to the project was high, with managers, clinicians, the in-house translation service and the IT department all ready to participate in both the development of the tool and its integration into Freedom from Torture systems. The first author was brought into the project as an external consultant with experience of developing tools to measure psychosocial wellbeing in many humanitarian settings, including northern Uganda, Democratic Republic of Congo, South Sudan and refugee camps in Kenya. In developing an outcome tool with Freedom from Torture, she followed the same processes as she would follow to develop a tool to measure psychosocial wellbeing in another culture; treating 'torture survivors' as a distinct (albeit heterogeneous) population, and beginning by trying to understand what psychosocial wellbeing and psychosocial distress meant to this population. ${ }^{6}$ Psychosocial wellbeing is a well-established concept in the humanitarian field. ${ }^{7,8}$ It refers to a person's capacity to realise their own abilities, cope with the stresses of life and be able to make a contribution to their community. Factors which play a part in this include human capacity (personal skills, abilities and strengths), along with the social networks and support available to an individual, and other elements such as culture and values.

As noted above, it is essential to develop a tool and process that is accepted by clients 
and clinicians, and understood by interpreters when they are present. Therefore, it was important that these groups were fully involved in the development of Freedom from Torture's clinical outcome tool, and the process by which it would be used. A slow, careful collaborative approach was used, with regular visits to each of the five Freedom from Torture centres to get input into the process from clients and clinicians, and ongoing discussions with clinicians by email as ideas started to come together. There were many opportunities for clinicians to have input into the process, and the first author took time to explain the decision-making at each stage, both in person during meetings at each centre, and in writing via email. The fears and concerns of clinicians about the nature of the final tool, and how the information gathered would be used, were acknowledged and addressed as much as possible. This approach took time, but was essential if the final tool and process were to meet the needs of clinicians and clients, and to be used effectively.

Following the initial conversations with clients and clinicians, it was clear that the following issues would be central to the development of a clinical outcome tool which would be appropriate for this context:

a) It would be 'torture specific', meaning that it would accurately reflect the experience of the Freedom from Torture client population. It would reflect the fact that distress is not only about torture experiences but also experiences of loss (due to forced migration or other factors such as death or disappearance of family members) and the client's experiences in the UK.

b) It would be appropriate for male and female adult torture survivors from different cultures and backgrounds. c) It would provide a valid and reliable measure of change in specified areas anticipated to be influenced by Freedom from Torture psychological rehabilitation services.

d) The tool would measure change in only those areas of psychosocial wellbeing which can be influenced by psychological therapy. Other issues which impact on wellbeing but cannot be influenced directly by psychological therapy (e.g. pain caused by torture or other physical factors) would not be included.

e) It would be based on understandings of psychosocial wellbeing as described by clients and clinicians, rather than on psychiatric diagnoses or symptom checklists.

f) It would also capture those factors expected to mediate the extent to which/ speed with which a client can make progress in psychological therapy (mediating factors).

g) It would primarily assess outcome, rather than process.

h) It would be relatively brief, so as not to be experienced as burdensome by either the client or the clinician. It would not, therefore, assess all aspects of wellbeing, but only those identified as most central by clinicians and clients.

i) The process of completing the outcome tool would be experienced as non-invasive and beneficial to the therapeutic process by both clinicians and clients. This would be essential not only to ensure that valid and reliable information was obtained, but also to avoid the use of the outcome tool being disruptive to the therapeutic relationship.

\section{Process}

The process of developing the outcome tool is summarised in Figure 1 (based on Jaranson and Quiroga, ${ }^{2}$ p113). 
Figure 1: Summary of the process of developing a clinical outcome tool for Freedom from Torture

PHASE I Review of literature and other information

PHASE II Discussions with clinicians $(n=29)$ and current/former service users $(n=23)$

PHASE III Consolidation of ideas regarding content and process Sharing consolidated ideas with clinicians for feedback

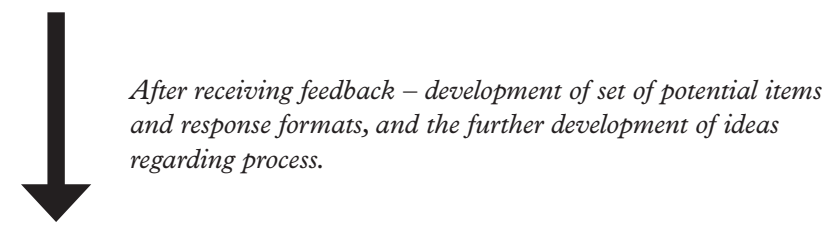

PHASE IV Sharing of revised ideas with clinicians for further feedback. Testing of potential items and response formats with clients Discussion of potential items with interpreters

PHASEV Development of draft clinical outcome tool ready for testing

PHASE VI Translation and back-translation

PHASEVII Pilot testing - 151 clients over a nine month period

PHASE VIII Sharing 'final' tool with clinicians, and minor changes made.

Guidance Notes developed in collaboration with clinicians.

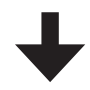

PHASE IX Translation

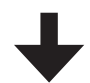

PHASE X Development of data entry system

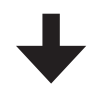

PHASE XI Roll out: collecting and recording data

Each phase is explained in more detail on the following pages. 


\section{Phase I}

Freedom from Torture's former Audit and Evaluation Team had gathered a considerable amount of literature relevant to the development of clinical outcome tools, so the first task was to review this information.

\section{Phase II}

The second phase was for the first author to visit each of the five Freedom from Torture centres to meet with clinicians, clients and former clients. The aims of these meetings were:

1. To increase understanding of the Freedom from Torture client population and the aims of individual therapeutic interventions.

2. To find out what clinicians and clients see as the key indicators of improvement for Freedom from Torture adult clients participating in individual therapy.

3. To find out what clinicians and clients see as the main mediating factors (i.e. those factors which affect the extent to which a client is able to make progress).

4. To identify clinicians' concerns about the development of a clinical outcome tool and the issues they feel need to be taken into account.

During these visits, the first author met with 23 clients and former clients, and with 29 clinicians.

\section{Phase III}

Following these discussions, and a continuing review of the literature in the field, the first author developed a consultation paper which summarised the information obtained and her thoughts on the outcome tool at that point. These included suggestions on the process of using the outcome tool, as well as suggestions for its content. This paper was shared with 21 clinicians, and the feedback received was used to develop a set of potential items and response formats, and to further develop ideas regarding the process by which the tool would be used.

\section{Phase IV}

The revised ideas were shared with clinicians, and the potential items and response formats tested out with clients. This involved meeting individually with 13 high-functioning clients selected by clinicians at two centres, trying out informally the potential items and five alternative response formats and asking the clients' opinions on their suitability. The potential items were also discussed with interpreters who spoke Arabic, Kurdish, Farsi, Turkish and French, to ensure that it was possible to translate the concepts accurately.

\section{Phase V}

This process of testing and discussion, together with further feedback from clinicians, led to the development of a draft clinical outcome tool ready for formal testing.

\section{Phase VI}

The draft version of the tool was professionally translated into each of the 15 languages in which it would be used, since reliance on verbal translation at the point of assessment would introduce a source of inconsistency. Freedom from Torture has an interpreting and translation service, and its manager coordinated the translation process, using translators who were familiar with Freedom from Torture's work.

Discussions with translators and translation agencies at Phase IV highlighted the subtle differences in meaning which can occur when a word is directly translated. The development team worked with translators, therefore, to ensure that the meaning of each item was captured in the 
translated version, rather than a literal translation, and a 'glossary' produced by the first author to explain the intended meaning of the items was shared with all translators and proof-readers. The translated tool was proof-read by a second translator and then back-translated into English by a third translator unfamiliar with the items. Discrepancies were discussed between the first author, the manager coordinating the translations and the senior clinician who commissioned the tool; feedback to the first translator was provided and the translated tool revised accordingly.

\section{Phase VII}

The draft tool consisted of 41 items, plus three 'feedback on process' items (more items than were intended to be included in the final version) and over a period of nine months it was administered to 151 clients by the clinicians who worked with them. Unless clinicians and clients had a language in common, this was done using a translated version of the tool with the support of interpreters. In addition to the data itself, clinicians and clients were asked to provide feedback on individual items, the response formats or any other aspect of the tool, and this feedback was also included in the analysis.

The clients with whom the tool was completed ranged in age from 17 to 59 (mean age $=35$ ). Almost three quarters were male (109 clients, $72.2 \%$ ) and one quarter female (42 clients, 27.8\%). 25 nationalities were represented, with the largest groups being Iranian and Sri Lankan. The tool was administered in 11 languages, with the highest proportions being administered in English, Farsi and Tamil (the two largest language groups within the Freedom from Torture client population at the time).

Each clinician involved in the pilot was requested to rate the severity of problems suffered by the client with whom they were completing the pilot tool using a four-point rating scale (Minimal problems - able to function well; Some problems - able to cope with many daily situations, even if with some difficulty; Severe problems - has difficulty managing day-to-day, but can carry out some key tasks; Extremely severe problems - barely able to function at all). This was partly to ensure that the tool had been tested with the full range of clients that it would eventually be used with, rather than only those with lower levels of psychosocial distress; and partly to enable the testing of items' ability to discriminate between clients with different severities of problems. The rating of the severity of the client's problems was a very rough assessment; the clinician was just asked to decide which 'quarter' the client would fit in, based on his/her experience of the client. The ratings are summarised below.

Table 1: Summary of the 'wellness' of clients included in the pilot study, according to clinician ratings

\begin{tabular}{lrr}
\hline Severity rating & N & \% \\
Minimal problems & 11 & 7.3 \\
Some problems & 89 & 58.9 \\
Severe problems & 33 & 21.9 \\
Extremely severe problems & 13 & 8.6 \\
Missing data & 5 & 3.3 \\
\hline
\end{tabular}

A range of statistical tests were carried out on the quantitative data, and thematic analysis conducted on the qualitative data. The main criteria taken into account when assessing the effectiveness of the elements of the draft tool were:

Acceptability/usability: This was assessed through feedback from clinicians, although any major problems with items had been identified during the initial testing process. 
Discriminant validity: Discriminant validity was evaluated by comparing the scores on the clinical outcome tool, and its various components, of clients with severe psychosocial problems (as rated by clinicians) with those functioning relatively well. We predicted that those identified as having severe psychosocial problems would score more highly than those with fewer problems.

Internal reliability: Tests of internal reliability were conducted to assess how the items related to each other. A close relationship between items would suggest that they are measuring a single underlying construct (e.g. psychosocial wellbeing), and that clients are responding to the items consistently. It may be that there is more than one underlying construct (e.g. emotional wellbeing and social wellbeing), which would not indicate a problem with the tool, but would be useful to know. If any item did not relate to the others, this could indicate a problem with that item.

Various statistical procedures were used to explore how well each quantitative item ('signs of wellbeing and distress') captured psychological wellbeing, and had the potential to be sensitive to change. Client and clinician feedback on how well items were understood was also reviewed. The procedures followed were:

1. Checked for endorsement frequency of items. If an item had a very high endorsement rate (indicating that a high proportion of clients experienced this 'very much') or a low endorsement rate (indicating that a high proportion of clients experienced this 'not at all'), then it should be eliminated. If everyone responded the same way to an item, regardless of the stage of change they were at, it would tell us very little about progress. Mean scores and standard deviations were reviewed, and two items showed a very low endorsement rate, so were not included in the final version.

2. Checked discrimination ability of items - which best discriminate between clients assessed by clinicians as having very severe problems, and those assessed as being relatively well. Those which discriminated best between these groups were more likely to be sensitive to change.

3. Checked correlations (Pearson) between items. It was not necessary to have two items measuring the same issue. A correlation between items which was significant at the $\mathrm{p}=.01$ level was considered to be high.

4. Checked internal consistency of items - how well each item correlated with other items and the total score. The factors considered here were whether the item correlated with the total score poorly (less than .2) and whether the reliability (Cronbach's alpha) of the scale would increase if this item was removed. These criteria applied to three items.

5. Conducted factor analysis to find out whether the tool assessed one single factor or a number of factors. Three factors with an eigenvalue of greater than 2.5 were identified (13 with an eigenvalue greater than 1). Six items did not load highly (i.e. greater than .4) onto any of the three main factors (varimax rotation).

Decisions were made about which items to eliminate and which to retain based on review of the whole pattern of findings, rather than on one single element of the analysis. This process resulted in a reduction from 41 items, plus three to obtain 'feedback on process' to 21 items plus one 'feedback on process'. One of the 21 items was a general question ('Given your answers to all these questions, how would you say things 
are in your life overall?); the other 20 were designed to measure different elements of wellbeing. The internal consistency of the final 20-item 'signs of wellbeing and distress' scale was good (Cronbach's alpha $=.83$ ), and it was able to distinguish between those with less severe and more severe problems. ${ }^{1}$

A visual aid (pictures of containers filled with varying levels of water) was used to illustrate the response options. Feedback from clinicians and clients indicated that this was generally helpful. One of the challenges experienced by some clinicians and clients was difficulty in focusing on the last week, so this issue was addressed in the Guidance Notes for clinicians. Another challenge was that some clients said their feelings changed many times in a day, and they could not rate how much they had experienced a particular feeling over a one week period. It should still be possible to generalise over one week, even if feelings are very changeable, so this was also addressed in the Guidance Notes.

Clinicians and clients reported that the concept of 'coping' was difficult for some to understand. Two questions were retained to assess clients' ability to cope, but the wording was changed to make it more concrete and readily understood. For example, 'Are you able to cope with distressing memories?' became 'Do distressing memories stop you from doing ordinary things?'

\section{Phase VIII}

The final version of the clinical outcome tool was developed and shared with Freedom from Torture teams at all five centres. Some minor changes were made following discussions with teams at the first two centres visited, and feedback from all centres was used to develop the Guidance Notes accompanying the tool.

Some clinicians felt that the name 'clinical outcome tool' was misleading, since the tool was intended to measure progress, as well as outcome. Therefore, it was renamed 'a measure of change and outcome' (MOCO).

\section{Description of final tool}

In general, the tool was designed to capture client change in key areas (as defined by clients and clinicians) in a valid and reliable way that does not disrupt (and may even enhance) the therapeutic relationship. Its design is outlined below. ${ }^{2}$

Background information: Minimal information is required because for each client the data from the MOCO will be linked to other data already held about the client on the Freedom from Torture information storage system.

Factors affecting wellbeing: A section in which the clinician and client explore in an open way the issues which have had the most impact on the client's wellbeing, either positively or negatively, over the last one month. Any issues raised by the client can be recorded in this section, along with whether they had a positive or negative impact. The ten issues most commonly mentioned in the pilot data are listed to facilitate clinician's recording of the information given by the client (see Table 2).

Signs of distress and wellbeing: 20 questions about the client's thoughts, feelings and behaviour over the previous one week. Clients are asked to indicate whether each thought/feeling/behaviour has affected them 'not at all', 'a little', 'quite a lot' or 'very much'. A visual aid illustrating each option with varying levels of water in a container is available if the client finds it helpful. Once

\footnotetext{
i A copy of the MOCO can be obtained from jvandi@ freedomfromtorture.org
} 
they have chosen the response that best reflects their feelings, the clinician invites them to say more about their answer. This qualitative aspect to the responses is important for two main reasons: it gives the client an opportunity to talk freely about the issues which are most affecting them if they choose to do so, which enables the process to have some therapeutic value; and it provides a check on whether the client has understood the question - this is particularly important since the questions are being asked in translation in many cases. If it emerges that certain questions are frequently misunderstood, it will be possible to revisit the translation, or the wording of the question (see Table 3).

Assessment of overall wellbeing: A single item asking clients to rate how 'things are in your life overall', using a four point scale (very difficult, difficult, good, very good). Again the client has the opportunity to explain their response.

Feedback on process: An open ended question at the end of the tool, asking the client to explain how they feel about the service they have received from Freedom from Torture over the previous three months. They are asked to explain what has been helpful to them, and what has not been so helpful. The clinician makes brief notes on their response.

Additional notes and feedback: A space for the clinician to record any notes and feedback. Any recurring issues raised in this section can be addressed when the tool and the process of administering it is reviewed.

The MOCO was designed to be administered during sessions, as a collaborative exercise between client and clinician, with the support of an interpreter when the
Table 2: The ten areas of life most commonly identified during the pilot study as having a positive or negative impact on clients' emotional wellbeing

1. Legal status/asylum process

2. Housing issues

3. Education issues

4. Social issues

5. Family issues

6. Physical health (excluding pain)

7. Physical pain

8. Financial issues

9. Purposeful activity/lack of activity

10. News from home

Table 3: The 20 'signs of distress and wellbeing' items included in the final version

How much have the following things affected you over the last ONE WEEK?

1. Forgetting to do things

2. Feeling very sad

3. Unwanted memories

4. Feeling tense, anxious or nervous

5. Difficulty with sleeping

6. Nightmares

7. Realising or being told by other people that you have done something you cannot remember.

8. Feeling as if you were actually back in the distressing situation

Now I'd like to ask you about some different types of experiences. Again, I'd like you to think about how much you have experienced each one over the last one week

9. Do distressing memories stop you from doing ordinary things?

10. Do you feel hopeless?

11. Do you feel that your soul is damaged?

12. Do you lose your temper over small things?

13. Do you feel ashamed?

14. Has talking with people felt like too much for you?

15. Have you been able to carry on with ordinary things when problems occur?

16. Have you felt lonely?

17. Have you lost interest in things?

18. Have you made plans to end your life?

19. Have your feelings gone dead?

20. How much do you trust other people? 
clinician and client do not share the same language. In this way, it could be used as a therapeutic tool, especially because it was designed to give clinicians and clients an opportunity to discuss the client's responses.

\section{Phase IX}

Although many items were the same as in the pilot version, there were some changes to the wording of questions, so these items went through the same process of translation, proof-reading and back-translation as the items included in the pilot version did. This phase proved particularly challenging in relation to engaging the services of translators available to do the back translations who were not familiar with the document. In this phase for instance, amongst the returned back translations, there was one that was identical to the original document in the source language (English), raising concerns about limited translation resources in some languages.

\section{Phase $X$}

Before it was possible to roll out the MOCO across the organisation, it was necessary to develop a system for data entry. Freedom from Torture uses a system called 'Daylight' to record information about clients, and the IT manager, in collaboration with the first author, adapted the Daylight system to allow data from the MOCO to be inputted directly by clinicians, and to be exported for analysis.

The advantage of the data being entered into the Daylight system is that it is integrated into Freedom from Torture's organisational processes. It is, therefore, possible to monitor clinicians' usage of the tool (e.g. to identify when it has not been completed as expected), and to include in the data analysis variables from other parts of Daylight, such as age, gender, nationality, forms of torture experienced, stage reached in the asylum application process, number of therapeutic sessions since last use of the tool, modality of therapeutic intervention employed, language used in the session and whether an interpreter was present.

\section{Phase XI}

The MOCO was formally rolled out across the organisation's five centres in April 2014. In the four centres outside London, all clinicians had been involved in the development of the tool so were very familiar with the format and how to administer it. Guidance notes were also available to assist clinicians in their administration of the tool. A much larger number of clinicians work in the London centre, so not all had been involved in the process of developing the tool. Clinicians in London were introduced to the tool and trained in its use during team meetings. Interpreters were briefed by practitioners about the use of the tool prior to their first contact with it in their language.

The use of the MOCO is now fully integrated into Freedom from Torture's systems. It is used as part of the initial client assessment, to be completed during the first few sessions. Since significant change can occur in the early stages of therapy, the tool is completed a second time after three months of therapy. Following this, it will be completed at six months and twelve months, with the final occasion taking place a couple of weeks before therapy ends.

Although it is desirable for the first assessment to take place as early as possible in the therapy, to avoid missing any positive changes that occur in the first few weeks, some clients are too distressed to engage with the process of completing the MOCO in the very early stages. In these cases, there is an option to give a clinician-rated score so that the high level of distress and any subsequent improvement can be captured. 
In order not to disrupt the therapeutic process, it is possible for the MOCO to be completed at any time during a three-week period at each point (the 3-month mark, 6-month, 12-month etc.), according to the judgement of the clinician.

\section{Next steps}

The MOCO developed by Freedom from Torture is still in the early stages of use. For example, we do not yet have a way of coding the qualitative data; this will be developed through thematic analysis of data as it becomes available. Despite this, some UK-based organisations working with similar populations have expressed interest in using the tool, and are trialling it with their clients. Freedom from Torture encourages this kind of collaborative working, and hopes to expand it once the MOCO has been in use for a longer period of time, and enough data has been collected to enable us to explore its psychometric properties.

A process of adapting the MOCO for use with young people is currently underway. The overall structure and some of the items are the same, but additional items have been included. The same process of development (discussion with clinicians and clients, etc.) was used for the development of this tool, and it is currently in the pilot stage.

It is important that the analysis of the data from the MOCO with adult clients is conducted not only in a way which is statistically accurate, but also in a way that provides information which will inform Freedom from Torture's policy and practice. The data analysis will, therefore, take place within a dynamic process of hypothesis generation, data analysis and presentation of findings, reflection, and further hypothesis generation, as described in Figure 2 below.

This process required two groups to be formed:

1. A 'hypothesis generating' group, made up of key people within the organisation, plus at least one member of the data analysis team.

2. A 'data analysis' group, made up of two or three people who work together to analyse the qualitative and quantitative data and present the findings.

Figure 2: The data analysis process

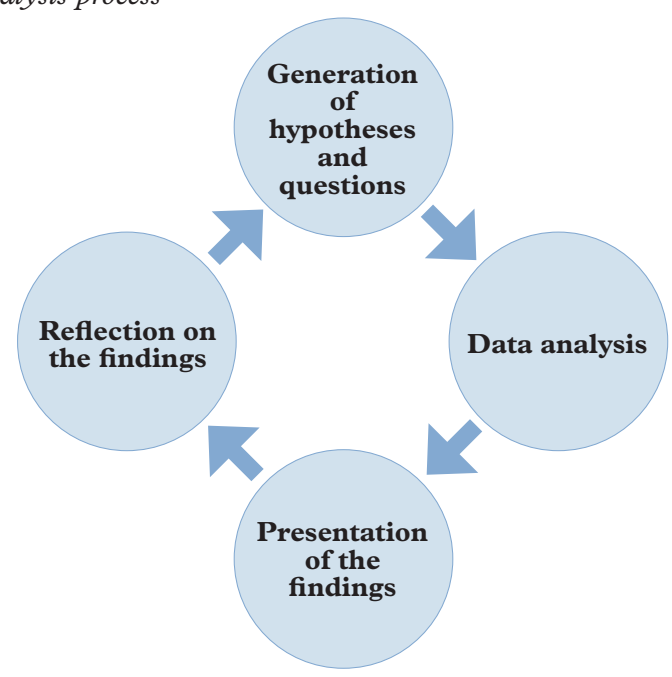


The first stage in the process, the generation of hypotheses and questions, focuses on identifying a set of questions which can be asked of the MOCO data. These questions should be those which can be answered with the type and amount of data available at the time, and which will deepen understanding of the Freedom from Torture client group and how they engage with therapy, in order to inform decisions about policy and practice, and/or for purposes of advocacy and communication.

Following agreement on the hypotheses and questions, the data analysis team conducts the analyses (stage 2). At stage 3, they present the findings in a report format, and also as a powerpoint presentation to be delivered to the hypotheses-generating group. The two groups together reflect on the findings (stage 4), and this leads to the development of a further set of hypotheses and questions to be addressed (stage 1). This process continues, enabling Freedom from Torture to ask new questions of the data as different issues emerge, and as the dataset increases.

The first phase of the analysis is about to begin at the time of writing, using this process. It will be possible to review and revise the planned 'cycle of analysis' once this is complete.

\section{Review}

Meetings were held in June 2015 with groups of clinicians, clients and interpreters to review their experiences of using the MOCO over the first year. The clinicians who shared their experiences identified a number of ways in which they had found using the tool beneficial:

- It is a helpful way of recognising progress that has been made.

- It can help clinicians to understand the client more fully; using the tool has sometimes enabled the client to disclose issues that were useful for both the client and clinician. These issues can then be revisited in later sessions if appropriate.

- It can be normalising, especially if the clinician explains that clients have had input into the development of the tool.

- Some clients found it helpful to have an 'overview' of their wellbeing in one session, even if the tool does not cover all issues which are relevant to wellbeing. It can be a 'containing' experience for clients who feel very fragmented.

- The frequency with which the tool is used feels manageable.

The clients who participated in the review found the tool helpful as a means of checking their 'progress or non-progress', and felt that the questions were good descriptions of their experiences. When they see that they have improved in certain areas, this is very positive for them.

Inevitably, there are aspects of using the tool which are challenging for clients, clinicians and interpreters.

The first time the MOCO tool is used with a client can be difficult, especially because the client is often highly distressed at that stage. Sometimes it is not possible to ask the questions in the first few sessions, either because the client is unable to focus sufficiently to answer them or because the process can be experienced as persecutory. In the first few weeks and months, a clinician's focus is on building a trusting relationship and enabling the client to feel safe; ethically there is no doubt that this must be the priority. At the same time, clinicians recognise that it is important to collect information at an early stage in order to reflect the extremely distressed state in which clients often arrive at Freedom from Torture.

One of the clients who participated in the 
review said that the first time his clinician went through the outcome tool with him, it brought back memories of his interrogation by security forces, which he found very difficult. This is where the skills of the therapist are essential: "I know him, he knows me and my weaknesses. He knows how to support me without leaving me mentally weak. He does his best to make me feel I'm not under torture. He's very clear, he shows affection. Do it in my own time, don't have to do it all now if you're not comfortable ... He says 'you're not forced' to answer that, so you feel he's someone sensitive to your issue" (Male client). One clinician described how she and the interpreter had to find a way of asking the questions in a conversational style, without either of them holding papers or making notes in front of the client, because he found this distressing: "We have to do it relationally, be creative and flexible" (Clinician).

Many of us find it difficult to be clear about how we have felt over the last one week, and not surprisingly, some clients have conceptual difficulties with rating their feelings. Again, considerable skill is required on the part of the clinician and interpreter to help them with this. This relates more to the first time the tool is used with someone; the second and third times are more straightforward.

Some questions are difficult to communicate the first time the tool is completed, especially in translation. The question 'Do you feel that your soul is damaged?' is extremely meaningful to some clients, especially those who have experienced the most extreme torture, but where it does not touch on someone's experience it can be difficult to understand. The clients who participated in the review felt that it was an important question, and that in some ways it was the ultimate test of someone's recovery.
It has not been possible to use the translations of the tool in a standardised way, as initially intended. There are not only many languages spoken within the Freedom from Torture population, but also many dialects. The interpreters said that they cannot use a standardised way of communicating the questions to clients, they have to adapt depending on the dialect spoken by the client, and even their level of education. Clearly this is not ideal, since if the questions are not asked in a standardised way it is more difficult to be confident about any changes and differences we observe. However, if the standardised questions are not well understood by clients, this would also prove to be a source of unreliability. The best way forward, it seems, is for all the interpreters to have a good understanding of the meaning of the questions, and to have a copy of the translated tool to refer to, but to communicate the meaning of each question in the way most appropriate to each client.

\section{Discussion}

The outcome tool developed by Freedom from Torture, and the process by which it will be used, has addressed some of the shortcomings of tools used in other outcome research (see reviews ${ }^{2,3,4}$ ). The characteristics of good research outlined earlier in this paper are useful in reviewing Freedom From Torture's measure of change and outcome.

- Measurement tools and approaches should consider the context within which torture survivors live. The Freedom from Torture tool includes a section on 'factors affecting wellbeing' which records in a brief format those issues which have the greatest impact (positive and negative) on clients' wellbeing. Over time, it will be possible to assess the ways in which these issues influence wellbeing.

- A broad range of outcomes should be consid- 
ered, and those which have the greatest meaning and relevance for the torture survivors themselves. The outcomes included in the MOCO were identified through an ongoing process of discussion with clinicians and clients. Those chosen for inclusion were the issues identified as having the greatest relevance for clients.

- Clinicians and other practitioners should be involved in the identification of outcomes and development of questions to be included in a measurement tool: Clinicians were fully involved in the development of both the tool itself, and the process by which it would be used.

- Translators should be involved in the development of questions to be included in a measurement tool: Potential items were discussed with Freedom from Torture interpreters before being included in the draft tool for piloting.

- Where the population is heterogeneous the sample size should be large enough to make comparisons: Since the MOCO will be used indefinitely with the entire population of adults engaged in individual therapy in the Freedom from Torture centres, large amounts of data will be collected over time. The outcome data can be analysed alongside assessment data and biographic information, so it will be possible to explore a wide range of important questions as the body of data increases, and to make comparisons between clients with different experiences and backgrounds.

- Outcomes should be assessed over a longer period than has been the case in previous studies: For each client, their psychosocial wellbeing will be assessed using the MOCO from their first contact with Freedom from Torture right through to when they end therapy, and at regular intervals in between.
The translation process is crucial in the development of clinical outcome tools intended for multilingual and multicultural populations, as noted by Patel, Kellezi \& Williams, ${ }^{4}$ and there are some very useful guidelines available to assist in ensuring that translation is conducted in a rigorous manner. ${ }^{9,} 10$

In order to achieve a cross-culturally validated instrument, Freedom from Torture took great care when translating the first measure of change and outcome tool piloting document into target languages. When commissioning the translations, Freedom from Torture held discussions with the translators emphasising the importance of focusing on the conceptual equivalence of a word or phrase, since a word-for-word translation (i.e. a literal translation) would not reflect the original meaning. Translators were instructed to consider the definition of the original term and attempt to translate it in the most relevant way. Additionally, they were provided with guidelines, including a glossary, written by the author of the tool. Similarly to the initial translation, emphasis when commissioning back translations was also on conceptual and cultural equivalence instead of linguistic or literal translation.

Due to the limited source of translators in the target languages, the translation process included 'forward translation' (Step $1^{9}$ ) by one translator rather than two. This enabled the first translation to be proof-read by a second translator before being back-translated by a third translator. Efforts were made to recruit qualified translators for all three stages, as strongly suggested by Sousa and Rojianasrirat, ${ }^{9}$ although the limited source of qualified translators in some languages created challenges in achieving this.

The adoption of blind back translations, as suggested by Sousa and Rojjanasrirat, ${ }^{9}$ 
was extremely helpful in identifying lack of clarity and discrepancies in words, phrases and sentences, and in highlighting uncertainty regarding the first translations.

Additionally, when the final pilot document was used with clients, they were asked to identify any words they did not understand, and words or expressions that they thought were unacceptable or offensive. The corrections made as a result of this process minimised the potential of clients misinterpreting questions, and greatly reduced clients' negative feedback about terms used in the tool.

\section{Limitations and challenges}

The MOCO is a tool designed for a specific purpose, and it is important to recognise both its strengths and its limitations. In order to avoid the MOCO becoming the focus of therapy, where the therapy is driven by the outcome tool, it is essential for both managers and clinicians to recognise and communicate the limitations of the tool, and that it is a tool, not 'the truth' about a client. It is useful for certain purposes, but does not tell the whole story about how clients change through their engagement with Freedom from Torture. Some of the limitations of the MOCO are outlined below, along with suggestions as to how these can be addressed, where possible.

The MOCO is designed only to assess the aspects of wellbeing identified as key by clinicians and clients. It is not designed to provide a full assessment of clients' wellbeing. There are, therefore, advantages to combining the use of the MOCO with other assessment tools.

There are a number of advantages to developing new instruments which are appropriate to a specific population, including the fact that the instrument can address the questions of particular interest to a programme; it can be developed in collaboration with the target population and other stakeholders; and can reflect issues of concern to the target population. ${ }^{11} \mathrm{~A}$ disadvantage, however, is that the use of different tools for different populations makes it difficult to make comparisons across different groups. This is a limitation which applies to the MOCO tool.

The question that many clinicians wanted an answer to is 'what is it about the way we work that helps our clients? Can we help clients more (or more quickly)?' To answer this question, it would be necessary to gather information about the approach an individual therapist takes with a particular client, and match this to client progress. The issue is complicated by the fact that, in many cases, clients receive a variety of services from Freedom from Torture, from a variety of personnel (therapist, physiotherapist, caseworker, doctor). With a large enough sample, it would be possible to evaluate which type of clients make the best progress with which approaches or combination of approaches, taking into account the characteristics of the client and his/her circumstances. However, it will be some time before Freedom from Torture has enough data to make this a possibility. In the meantime, clients will be asked what has helped them most over the last three months, and they will have an opportunity to identify any aspects of the Freedom from Torture service.

In order to be sure that it is a client's engagement with Freedom from Torture, rather than something else, that has contributed to any observed change we would need a comparison group of people with similar characteristics to Freedom from Torture clients, but who are not accessing Freedom from Torture therapy. Whilst this is not possible, Jaranson and 
Quiroga ${ }^{2}$ suggest that a comparison design can be used in these situations, where groups participating in different types of psychotherapies are compared (taking into account any differences in the characteristics of these groups, or other potential confounding variables).

Some clinicians (but not all) felt that their clients are unlikely to be comfortable telling them what they have not found helpful about the service they have received from Freedom from Torture, so this aspect of the MOCO tool would yield potentially less useful information. The feedback from clients on the service they have received from Freedom from Torture is an important part of the measure of change and outcome tool, but it should not be seen as the only 'truth'; it provides one perspective to be combined with others. Clients should have a variety of ways to give feedback to Freedom from Torture, including anonymous written feedback, and via clients' groups.

A concern expressed by some clinicians was, 'if clients don't improve, what does that tell us?' With all groups engaging with therapy, but particularly with the population Freedom from Torture work with, there are many external factors which influence a client's psychosocial wellbeing and whether they are able to engage with therapy. To some extent, we will be able to capture these external factors in the clinical outcome tool. However, it will also be necessary to be sensitive during analysis to the more subtle indicators of the positive impact of therapy. For example, if a client consistently scores highly over time on the item relating to suicidal intention, yet still keeps coming to sessions, this reflects a positive outcome. As some clinicians pointed out, simply staying alive is an achievement for some clients.
The issue of physical symptoms of distress was a challenging one. Whilst headaches, bodily aches and pains and other somatic signs can be an indicator of psychological distress, they may also be due to physical illness. Since it will be impossible to distinguish between the two in a tool such as this, no item relating to physical pain was included in the 'signs of wellbeing and distress' section of the MOCO.

Finally, clinicians noted that some clients might be motivated not to show any improvement until after their asylum claim is successful, due to the fear that if they are 'well' they may not be seen as requiring refugee status. As information accumulates, it will be possible to develop our understanding of how the asylum process affects psychosocial wellbeing. Clinicians gave as many anecdotal accounts of clients' wellbeing deteriorating once they got refugee status as accounts of it improving. This is an issue we will be able to understand better once data is available.

\section{Conclusions}

"We should all aim to start somewhere, and this may mean working with what we have, and what we are able to do in our unique country settings, and with the available resources and skills ... However, starting somewhere may also require that there be a shift in organizational culture to enable data collection and research, including outcome evaluation, so that traditional divisions and suspicions can be minimised, and there can be shared ownership within and across organisations/centres in seeing research as essential to developing context relevant, culturally-appropriate and effective rehabilitation services"(p145). ${ }^{5}$

Although there are undoubtedly imperfections in the MOCO developed by Freedom from Torture, and in the process by 
which it is being implemented, we have 'started somewhere', and have met the criteria referred to earlier in this paper set by a task force of senior clinicians for torture rehabilitation centres. ${ }^{2}$ Measuring outcomes is now an integral part of the care offered by Freedom from Torture clinicians to adult clients engaging in individual therapy. ${ }^{\mathrm{i}}$ Assessment is integrated into the daily routine of the Freedom from Torture Centres; it is not conducted as a separate research programme. It is perceived by Freedom from Torture trustees, management and clinicians as a long-term process, which will provide information over time to enable the organisation to identify those aspects of their services which have a positive impact on the various client groups they work with. Processes are in place to ensure that there is regular questioning of the outcome data, consisting of identifying key questions, analysing the data and reporting the findings, and using this information to improve the quality of care offered by Freedom from Torture.

The content of the MOCO will continue to be reviewed, and the processes used to collect and record the data, and will make use of it to improve services. Now that data is being collected and the first phase of analysis is underway, the work has just begun.

\section{Acknowledgements}

Thanks are due to Clarisa Carvalho (Interpreting Service Manager) for her work managing the translation of the measure of change and outcome, and her contribution to this paper. We would also like to acknowledge the efforts of all Freedom from Torture Clinical Managers, clinicians, clients and interpreters in the development of the MOCO, and in its ongoing use. Particular thanks are due to
Dr Nimisha Patel and her team, formerly of Freedom from Torture (The Medical Foundation) for the comprehensive review of literature relating to outcome measurement in this field which laid the foundations for this work. 


\section{References}

1. Patel N, Williams C de C A. Monitoring and evaluation of rehabilitation services for torture survivors: Handbook for service providers. London: ICHHR; 2014.

2. Jaranson JM, Quiroga J. Evaluating the services of torture rehabilitation programmes: History and recommendations. Torture. 2011;21(2):98140 .

3. McFarlane C.A, Kaplan I. Evidence-based psychological interventions for adult survivors of torture and trauma: A 30-year review. Transcult Psychiatry. 2012 Jul;49(3-4):539-67.

4. Patel N, Kellezi B, Williams C de C A. Psychological, social and welfare interventions for psychological health and well-being of torture survivors. Cochrane Database of Systematic Reviews 2014, Issue 11. Art. No.: CD009317. DOI: 10.1002/14651858.CD009317.pub2.

5. Montgomery E, Patel N. Torture rehabilitation: Reflections on treatment outcome studies. Torture. 2011;21(2):141-5.

6. Bolton P, Michalopoulos L, Ahmed AMA, Murray LK, Bass J. The mental health and psychosocial problems of survivors of torture and genocide in Kurdistan, Northern Iraq: A brief qualitative study. Torture. 2013;23(1):1-14.

7. Psychosocial Working Group. Psychosocial intervention in complex emergencies: A Conceptual Framework. Edinburgh: Psychosocial Working Group; 2003.

8. Inter-Agency Standing Committee (IASC), IASC Guidelines on Mental Health and Psychosocial Support in Emergencies. Geneva: IASC; 2007.

9. Sousa VD, Rojjanasrirat W. Translation, adaptation and validation of instruments or scales for use in cross-cultural health care research: a clear and user-friendly guideline. J Eval Clin Pract. 2011 Apr;17(2):268-74

10. World Health Organisation, Process of translation and adaption of instruments [Internet] [cited Mar 26] Available from: http://www.who.int/substance_abuse/research_tools/translation/en/

11. Hubbard J, Miller KE. Evaluating Ecological Mental Health Interventions in Refugee Communities. In Miller K, Rasco L editors. The Mental Health of Refugees. New Jersey: Lawrence Erlbaum; 2004. P. 337-374. 